\title{
Main weaving nozzles - ejectors
}

\author{
Karel Adamek ${ }^{1}$, Jan Kolar ${ }^{1}$, Jaroslav Pelant ${ }^{2}$, Jan Simak ${ }^{2}$, Simone Gramsch ${ }^{3}$ \\ ${ }^{1}$ VÚTS - Centre of Machinery Research, Liberec, Czech Rep \\ ${ }^{2}$ VZLÚ - Aeronautical Research and Testing Institute, Praha, Czech Rep \\ ${ }^{3}$ Fraunhofer ITWM, Kaiserslautern, Germany
}

\section{Email address:}

karel.adamek@vuts.cz (K. Adamek), jan.kolar@vuts.cz (J. Kolar), pelant@ vzlu.cz (J. Pelant), simak@ vzlu.cz (J. Simak), gramsch@itwm.fraunhofer.de (S. Gramsch)

\section{To cite this article:}

Karel Adamek, Jan Kolar, Jaroslav Pelant, Jan Simak, Simone Gramsch. Main Weaving Nozzles - Ejectors. International Journal of Mechanical Engineering and Applications. Special Issue: Moving Forward to Monitory Democracy: Citizens Engagement in Scrutinizing Election Process in Indonesian 2014 General Election. Vol. 3, No. 1-1, 2015, pp. 5-12. doi: 10.11648/j.ijmea.s.2015030101.12

\begin{abstract}
The paper deals both with numerical flow simulations and experiments in ejectors, used here first of all as main weaving nozzles, further as a source of air suction, etc. The problem of high noise level of expanded air flow is discussed, too.
\end{abstract}

Keywords: Numerical Flow Simulation, Ejector, Weaving Nozzle, Aerodynamic Noise

\section{Introduction}

Air jet weft insertion is the most powerful system among loom types. For the first imagination we can state that a standard air jet loom of a weaving width of $1.9 \mathrm{~m}$ operates of about 800-1000 RPM, with a corresponding time of each working period of about 75 to 60 milliseconds. Approximately, one half of it is used for the weft yarn transport through the open system of warp yarn to create the cloth. With the average value of weft transport, velocity reaches of about 50 to $60 \mathrm{~m} / \mathrm{s}$ (180 to $210 \mathrm{~km} /$ hour) with periodical arising and ceasing in every working cycle.

The consumption of compressed energy is an indispensable item in the total energy balance of the loom. The presented results of numerical modeling were focused on the highest exploitation of the compressed energy for weft transport. The main contributions to this topic are for instance [1] to [14]. Some interesting results are presented below.

\section{Numerical Simulations}

\subsection{Cylindrical Mixing Tube}

At the following results of modeling, it is observable the influence of different shapes of mixing tube at the flow field image. Usually used air pressure from 300 to $600 \mathrm{kPa}$ causes overcritical flow, so the presented flow fields contains isolines of Mach number. The critical value of $\mathrm{Ma}=1$ arises in the narrowest section of the primary inlet. In the cylindrical tube after Fig. 2-1, there arise several flow reflections invoked by the inlet shape in the mixing tube. The consequence of it is a non-uniform lengthwise velocity profile, which directly influences a non-uniform flow force affecting on the weft yarn (the aerodynamic force is proportional to the product of air density and the second power of the air velocity). The shape of weft yarn - the linear body of very small bending stiffness inserted in such a non-uniform flow field, is not straight and some problems can arise at the weft picking.

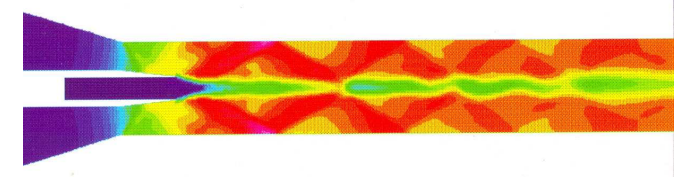

Figure 2-1. Isolines of the Mach number in cylindrical mixing tube.

\subsection{Divergent Mixing Tube}

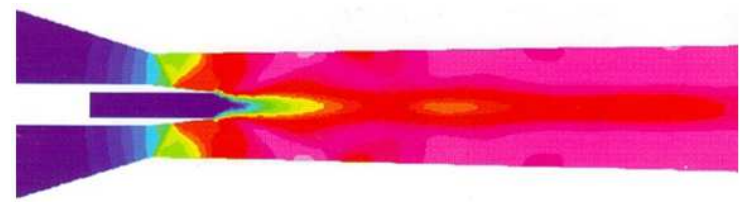

Figure 2-2. Isolines of the Mach number in divergent mixing tube

The randomly designed slightly divergent shape of mixing tube after Fig. 2-2 is more suitable for existing overcritical expansion, the flow field is more uniform here and of higher 
values. Generally, it is necessary to take in account a more complicated manufacturing of such a long and slightly conical shape, in comparison with the previous cylindrical tube.

The results of the presented modeling can be used as a source for the following evaluation. As an illustration, we can observe the saw-tooth graph in Fig. 2-3, presenting velocity profiles along the nozzle axis from the previous cases of cylindrical (Fig. 2-1) and divergent (Fig. 2-2) mixing tube. Corresponding density profiles should have an inverted character, given by Bernoulli's and gas state equations. Individual points of the graph present the value in individual mesh element of the above mentioned numerical model.

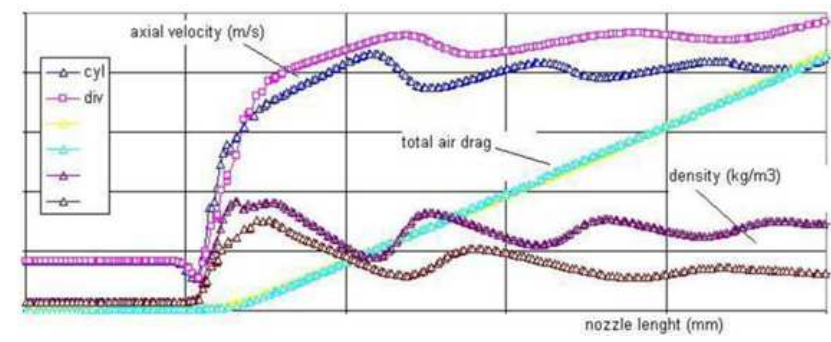

Figure 2-3. Total air drag as evaluation of previous flow fields

The corresponding air drag force caused by the presented flow field on an ideal weft body positioned along the nozzle axis is proportional to the product of air density and the second power of its velocity. Total air drag on total weft length (measured from zero to instantaneous position at the nozzle axis) is equal to the sum of individual values. By comparing profiles of various nozzle designs it is possible to choose the optimum one. The presented graph shows that conditions for expansion are more suitable in divergent shape - velocity values are higher, density values are smaller, oscillating character of both is smaller. But the influence of individual differences on total air drag is here negligible.

\subsection{Stepped Mixing Tube}

In order to get a higher and defined air drag force it is suitable to elongate the mixing tube, but by the same way, the flow resistance is increasing, too, and some part of primary air could blow back out through the suction (left) inlet. In this way, the suction effect, necessary for simple weft feeding after its breakage, is missing. It is possible to decrease this resistance by increasing the mixing tube diameter at its end, for instance after Fig. 2-4 - the tube total length is here twice greater in comparison with the previous cases.

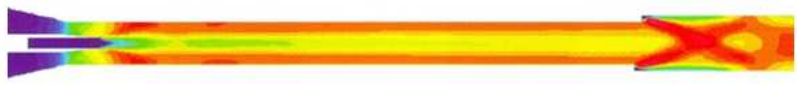

Figure 2-4. Isolines of Mach number in stepped mixing tube (double length)

In addition, another positive effect was reached here - the velocity maximum value is situated at the enlarged end area of the mixing tube, so we can state that linear body with negligible bending stiffness it pulled on its tip and not pushed by the flow field from previous Fig. 2-1 with maximum value at the (left) beginning of the mixing tube. By this manner, the stable weft form and position at the nozzle outlet is reached. The idea was verified by real weaving trials, too.

\subsection{Insufficient Suction Effect}

The optimum flow through an ejector similar to the previous Figures is influenced by the mutual ratios of several its dimensions and shapes, too. In Fig. 2-5, it is presented a detail of vector field in the mouth of the central weft tube for a specific design case where the nozzle main dimensions were designed unsuitably (for instance when the mixing tube is too long or convergent or the compressed air inlet is too large in relation to the mixing tube cross section, etc.).

In such a case, a part of the inlet driving air flow is flowing back and blows out through the central suction tube. The weft cannot be sucked in, this design case is not suitable for practical use.

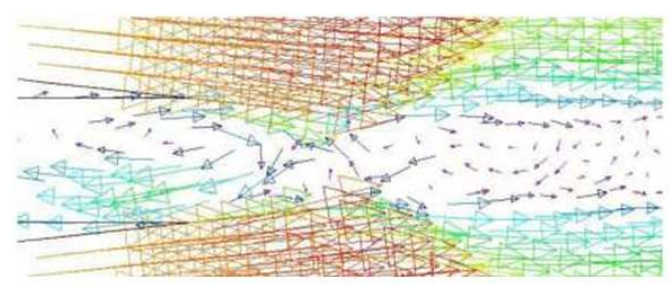

Figure 2-5. Example of insufficient suction effects

\subsection{Split Expansion at Nozzle Inlet}

The previous Figures use an optimum shape of the inlet part with the fluent and undisturbed inlet of primary air. The inlet part of standard main nozzle types is usually designed after Fig. 2-6 as several axial holes in the wall between the air tank (left) and the inlet part of the ejector (right).

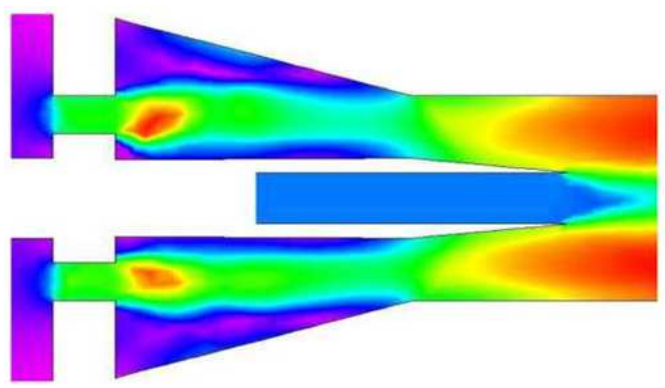

Figure 2-6. Split expansion at nozzle inlet

It is evident that air expansion is divided here into two parts. Hereby, the air flow after the first part of expansion is going into an interspace, where the flow cannot influence the weft yarn. The design of the main nozzle like this gives not so high air drag as the design after the previous Fig. 2-1 and not so high weaving output in $\mathrm{m} / \mathrm{s}$ of weft, too. On the other side, it is necessary to notice that the air drag value of the nozzle after Fig. 2-1 is so high that some spun weft materials of decreased strength can be broken by acceleration in such a nozzle. 


\subsection{Free Flow from Main Nozzle}

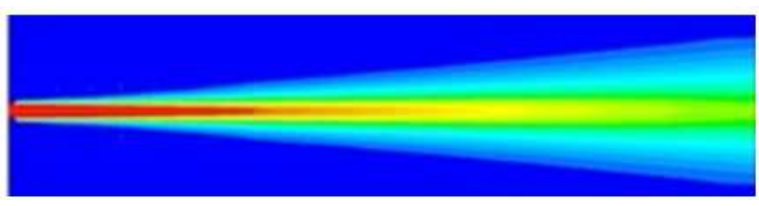

Figure 2-7. Isolines of Mach number - lower pressure ratio

The general image of flow field after the outlet from the mixing tube is described by standard equations of free flow propagation, expressing the progressive enlarging and ceasing the free flow area by the interaction with the unmoving surroundings.

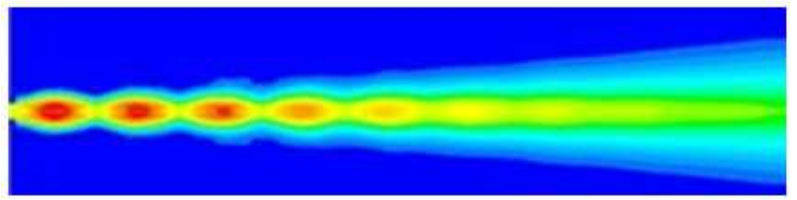

Figure 2-8. Isolines of Mach number-overcritical pressure ratio

As an illustration, Fig. 2-7 presents a typical flow field of free flow from an ejector. The usually used overcritical pressure ratios in Fig. 2-8 are the reason of typical shock effects in the free flow characterized by changing of expansion and compression areas until the free flow is dissipated enough. By reason of the used large mesh elements, the shape of expansion/compression areas in free flow are not so sharp as usual. To get the maximum resolution, the used scales ranges of Fig. 2-7 and Fig. 2-8 are different.

A fully analogous image of the flow field is valid for another nozzle shape, too.

\subsection{Dual Main Nozzle}

Another possibility how to increase the total force action of air on weft yarn could be the dual main nozzle with flow field after Fig. 2-8.

It is clear that the total air drag from two identical main nozzles arranged ,in tandem“ is not equal to the double air drag of one nozzle because practically it is not possible to tune the force effects of both nozzles. After the weft acceleration by the expanding air flow in the first nozzle, the velocity in the second one should be much higher to get the same force effect on the second nozzle. But the flow velocity inside the nozzle is limited by the practically used values of inlet pressure and by the nozzle inner shape, too.

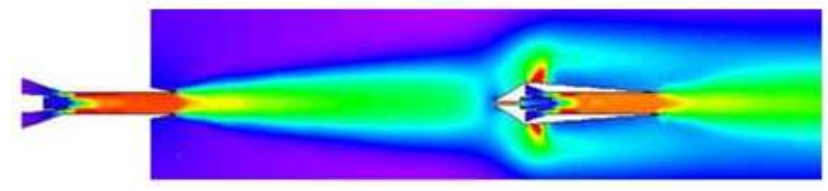

Figure 2-9. Isolines of Mach number - two main nozzles in "tandem"

\subsection{Aerodynamic Noise}

The high noise level during overcritical air expansion is caused by high pressure gradients in the expanding flow. Thus, such pressure fields below can be used as the first estimation of the noise level. The used commercial software offers the direct calculations of such acoustic fields of aerodynamic cases, but actually as flows of constant density, only, it means at the relative low velocities. Typically, there are the velocities of some tenths of $\mathrm{m} / \mathrm{s}$, as for instance the blowing around a car body. The numerical simulation of acoustic field for compressible flows, typically the (over)critical air expansion, for instance see Fig. 2-8 above, is not available now, some advances with such a solution are in progress now [15], [16], [17].

One simple case of blowing gun, solved by an alternative method of numerical flow simulation, is presented here. Fig. 2-10 presents isobars of overcritical free flow of expanded air behind the standard blowing gun with the removed outlet part of small diameter. The flow through the nozzle is much higher than the usual air flow, which except its own noise from the expansion creates the secondary very intensive noise by the reflection from the structured blown surface.

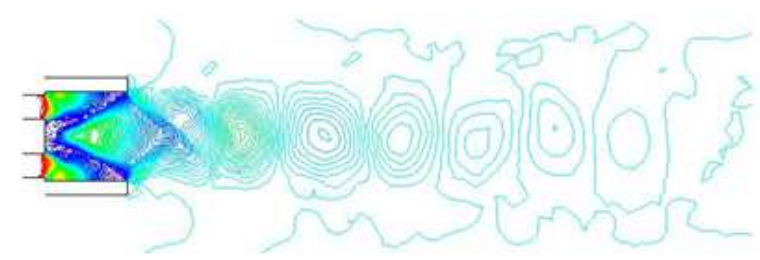

Figure 2-10. Areas of compressions and expansions in outlet of overcritical air flow.

Alternating areas of expansions and compressions are the sources of very intensive noise. Using reduced outlet with smaller outlet cross section the noise level was decreased.

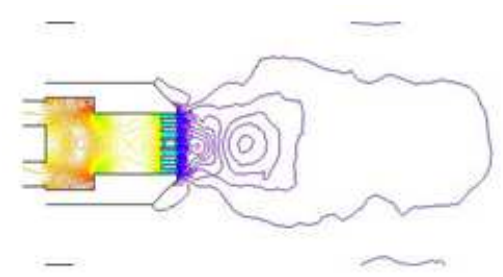

Figure 2-11. Small pressure gradients at the outlet of multi-hole nozzle

After the field of isobars for the case of multi-hole outlet after the Fig. 2-11 it is possible to judge that the noise level of such adaptation is much lower.

It is necessary to decide among the lower output of lower noise level and lower air consumption or hearing protection of the operational staff.

The last Fig. 2-12 presents the experimentally evaluated isolines of acoustic field of overcritical air flow from such blowing gun as above (outlet $=$ red dot). 


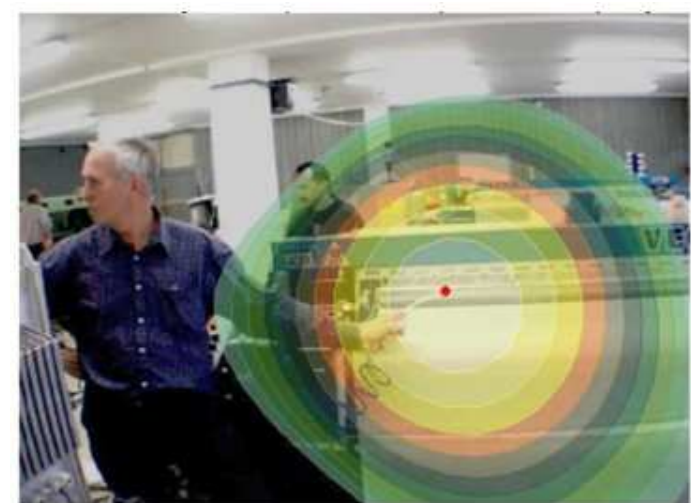

Figure 2-12. Experimentally evaluated isolines of acoustic field of overcritical air flow from blowing gun

\section{Experiments}

\subsection{Controlled Suction of Ejector}

In the graph in Fig. 3-1, there is presented the result of the experiment - measured suction $(\mathrm{kPa})$ in the inlet of secondary medium - for the nozzle /ejector equipped with an adjustable inlet of the primary medium.

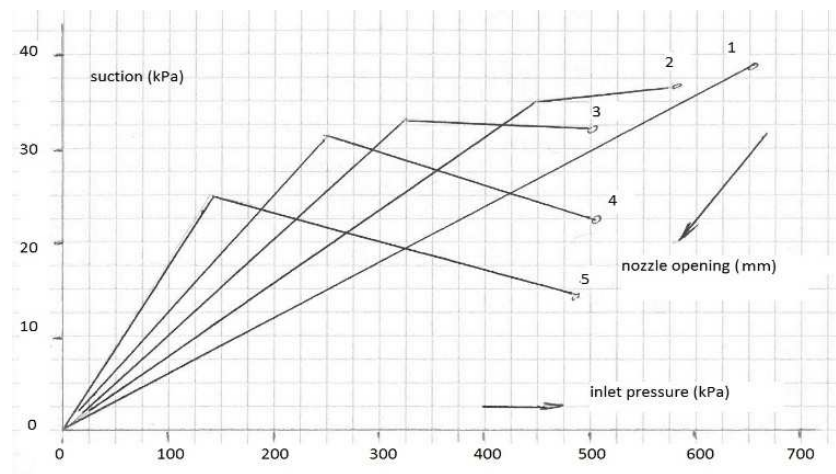

Figure 3-1. Suction effect $(k P a)$ of an adjustable ejector

The principle is similar to the following illustrations of flow fields, where it is possible to change the inlet cross-section of the primary medium by the axial shifting of the central tube. The second variable there is the pressure of the primary medium.

At the smallest nozzle opening (curve No. 1), the suction in the secondary inlet $(\mathrm{kPa}$ - vertical axis) is practically proportional to the pressure in the primary inlet $(\mathrm{kPa}-$ horizontal axis). With an increased nozzle opening (curve No. 2 ), the suction in the secondary inlet reaches its steady maximum, at the highest inlet pressures it begins to decrease (curve No. 3) respectively. This trend is continuing at higher nozzle openings (curve No. 4, 5), but the maximum suction value is decreasing gradually and is shifting to the lower primary pressures.

The larger nozzle signifies the larger consumption of the primary medium, so the operation is not so efficient. The course of the so-called ejection coefficient, it means the ratio of the secondary flow to the primary one, is very similar to the above mentioned relation, the typical value is 0.15 approx., but not more than 0.2. This value is many times smaller comparing with so-called wall-effect-nozzle, but ejector is able to develop the considerable outlet pressure or suction effect, too.

\subsection{Tensile Force}

The tensile force can be simply calculated after the well-known formula for the force effect of the flow on the rigid body

$$
\mathrm{F}[\mathrm{N}]=\mathrm{cx} \cdot \mathrm{S} \cdot \rho / 2 \cdot(\mathrm{w}-\mathrm{u})^{2}
$$

where

$\mathrm{c}_{\mathrm{x}}(-) \quad$ drag coefficient for given body shape

$\mathrm{S}=\pi \cdot \mathrm{d} \cdot \mathrm{dx}\left(\mathrm{m}^{2}\right) \quad$ surface of flowed body

$\rho\left(\mathrm{kg} / \mathrm{m}^{3}\right) \quad$ medium density

$\mathrm{w}(\mathrm{m} / \mathrm{s}) \quad$ medium velocity

$\mathrm{u}(\mathrm{m} / \mathrm{s}) \quad$ flowed body velocity.

The power carried from the medium onto the transported body is

$$
\mathrm{P}[\mathrm{W}]=\mathrm{F} \cdot \mathrm{u}
$$

so it depends on the momentary relation of body velocity and medium velocity. If the body velocity (u) is either equal to zero or equal to the flow velocity (w), the transferred power is equal to zero.

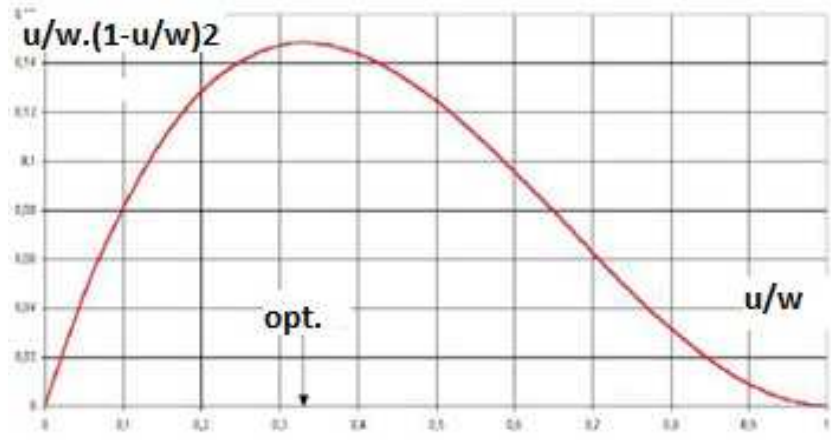

Figure 3-2. Optimum tensile force of ejector - theory

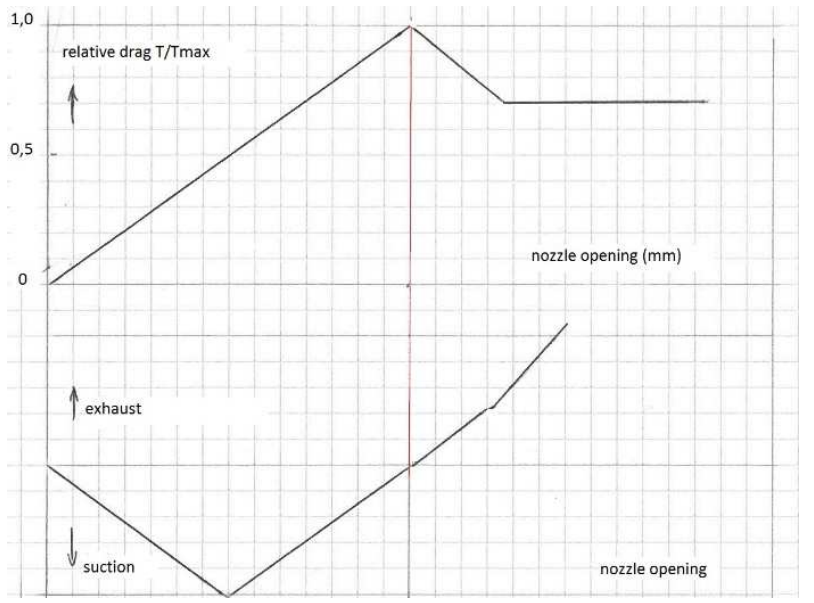

Figure 3-3. Relative tensile force (T/Tmax) (up) and suction effect of measured ejectors (down) 
The maximum transfer of the power from the flow onto the body comes at the ratio $\mathrm{u} / \mathrm{w}=1 / 3$, see the graph in Fig. 3-2. The general solution of the interaction between the flow and an elastic drifted body is much more complicated, in comparison with the only solution of the flow field.

Fig. 3-3 presents the schematic result of experiment with several types of real weaving nozzles, their designs are corresponding in principle to the above discussed ejectors [18]. Measuring was realized for several values of inlet air pressure, therefore, the results are presented as relative tensile forces T/Tmax. in dependence on the nozzle opening (i.e. on the air flow).

At the same time, the suction ability was measured, too from the operational point of view it is important to drag the broken yarn in the nozzle whenever. The practical working point of the nozzle, it means the nozzle opening, should be a little before the point when the nozzle suction is passing into exhaust. At the same working point the tensile force is maximum.

The really measured characteristics are in good coincidence with the theoretically simulated ones, see Fig. 3-1.

Remark - for the measuring of the tensile force it was necessary to use a thick nylon fishing-line, able to last the intensive acting of the air flow. At static measuring (standing yarn in the air flow), the used yarn or thin fishing-line was broken very quickly.

\subsection{Air drag Simulation}

At the following Fig. 3-4, there is presented the observed shape of the ejector and its velocity field. For a better depicting of details, there is displayed the inlet part with the beginning of mixing tube and the end of mixing tube, just before the outlet, only. In the absent middle part, the velocity field is constant. As variables there are chosen the pressure in the inlet (300-400-500 kPa) and the shape of the mixing chamber after Par. 2 above (cylindrical 100\%, divergent $110 \%$ and convergent $90 \%$ ), together 9 combinations.

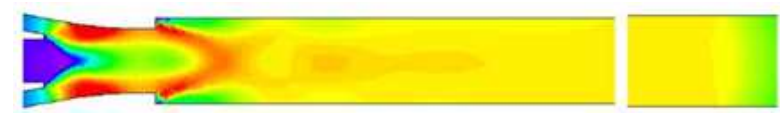

Figure 3-4. Velocity field of one monitored ejector

Remark: the curve marked for instance „105“ means the divergent channel $110 \%$ at inlet pressure of $500 \mathrm{kPa}$, etc.

The evaluated numerical simulations are presented as velocity profiles $\mathrm{w}(\mathrm{m} / \mathrm{s})$ (Fig. 3-5) and density $\rho\left(\mathrm{kg} / \mathrm{m}^{3}\right)$ respectively (Fig. 3-6). It is well visible the expanded flow for lower pressures in the inlet and the shock compression, appearing at higher pressures in various points along the ejector axis or as far as in the nozzle outlet.

Three typical modes are visible there: subcritical, or not fully expanded, characterized by low velocities and high densities (blue lines), transitional (yelow line) and overcritical, or fully expanded, characterized by high velocities and low densities (red + green lines).

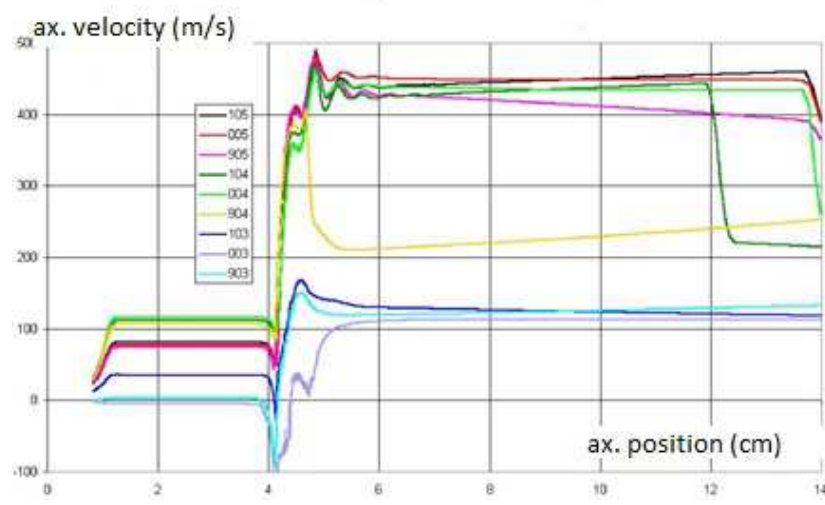

Figure 3-5. Velocity $(\mathrm{m} / \mathrm{s})$ profiles along ejector axis $(\mathrm{m})$

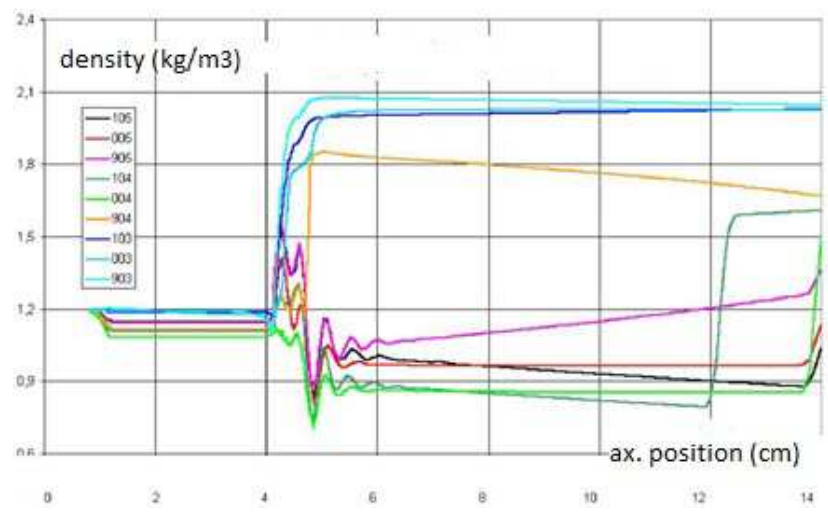

Figure 3-6. Density $\left(\mathrm{kg} / \mathrm{m}^{3}\right)$ profiles along ejector axis $(\mathrm{m})$

From those quantities it is possible to determine the total static air drag, proportional to the flow dynamic pressure $\rho / 2 . w^{2}(\mathrm{~Pa})$. The result of another solved case (see Fig. 2-3 above) shows that oscillating both velocity and density along a relative short part of the nozzle axis have not any influence on the total drag of the nozzle.

From the summary graph of tensile force for various inlet pressures after Fig. 3-7 it is visible that the maximum tensile force is reached for a slightly convergent mixing tube (the cross-section ratio inlet/outlet $=0,9$ approx.), but with a practical problem of long and slow taper manufacturing.

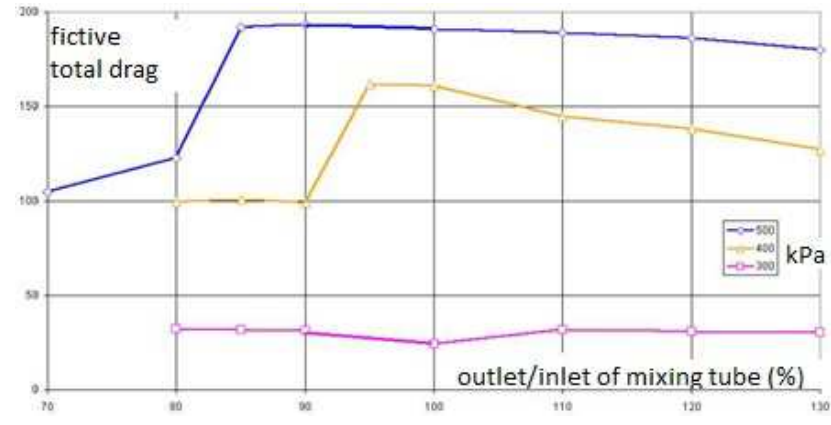

Figure 3-7. Total air drag for convergent/divergent mixing chambers

\subsection{Recirculation Bubble}

At the beginning of the mixing tube, there arises at certain geometrical conditions the so-called recirculation bubble, described in [20]. Its position, intensity and length are 
changing after the relations of cross-sections and lengths of the mixing tube. The inlet pressure has some influence, too. This phenomenon is explained in such a way that in the area of driving flow expansion, there arise so high velocity values that the suction effect at the end of the central tube causes some backflow of the main flow along the axis of mixing tube. Probably, it could be any kind of flow loss, which should be prevented.

For instance, in the ejector shape after the previous paragraph, used for the study of both the mixing tube shape and the inlet pressure on the tensile force, the recirculation bubble is none, but in the following presented case [21], [22], the recirculation bubble is short and very intensive. This area can be characterized as a deformed torus with backflow, well visible in the directional field Fig. 3-8.

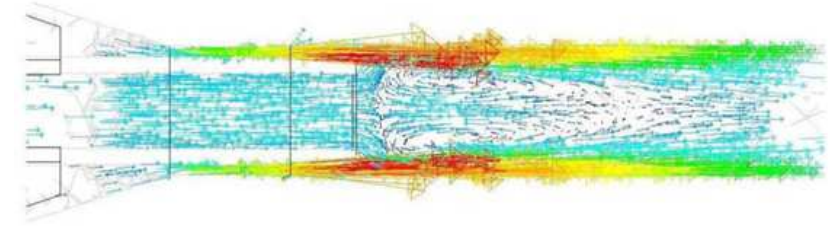

Figure 3-8. Directional field in the area of recirculation bubble

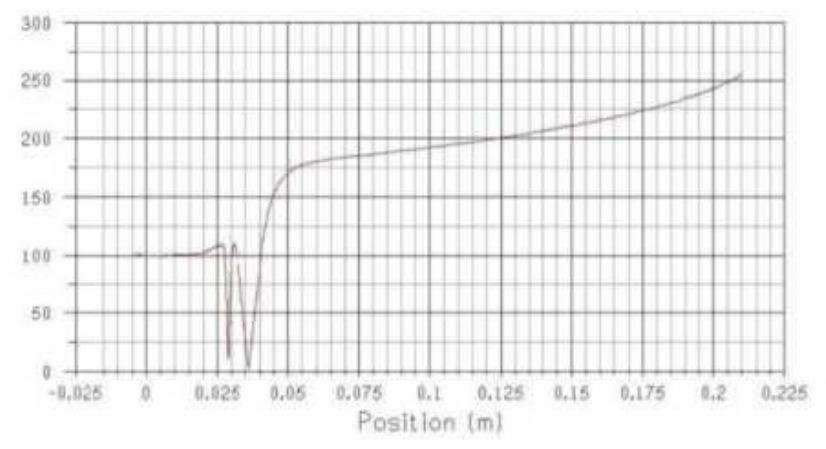

Figure 3-9. Axial velocity profile $(\mathrm{m} / \mathrm{s})$ along ejector axis $(\mathrm{m})$

In the profile of velocity field along the ejector axis, (Fig. 3-9) there is visible the double jumping deceleration in the fluently increasing velocity profile. In the profile of the cross (radial) velocity (Fig. 3-10), it is visible several alternating oscillations, too.

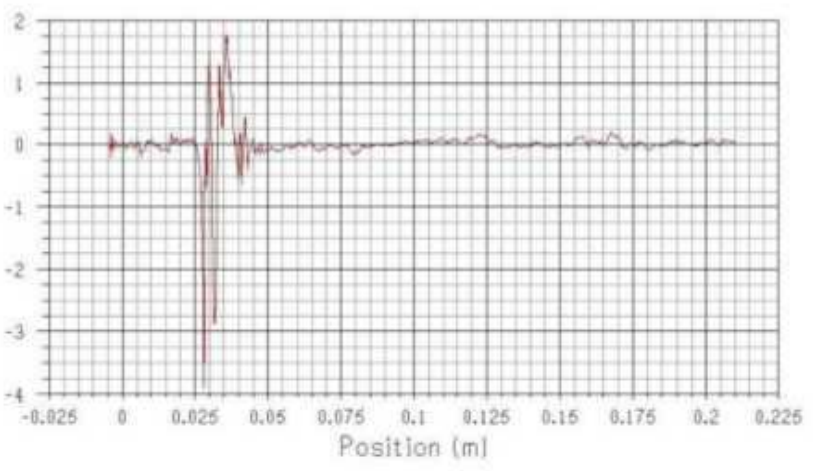

Figure 3-10. Radial velocity profile $(\mathrm{m} / \mathrm{s})$ along ejector axis $(\mathrm{m})$

In Fig. 3-11, there is presented the adverse effect of such a phenomenon: During a long-time weaving of aggressive glass yarns, the individual fibers of the yarn are in the area of recirculation bubble separating or swinging out from the nozzle axis to the mixing tube wall and they erode the inner surface of the mixing tube in such a way that after some operational period the tube is fully destroyed. We can see the inside view on such a destroyed mixing tube together with the eroded neighboring area, too.

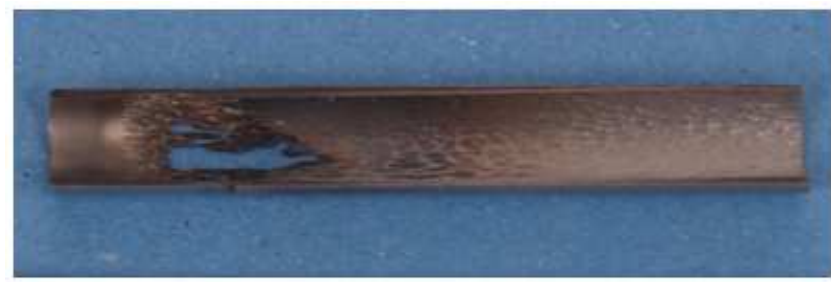

Figure 3-11. Erosion of the mixing tube wall

The axial position of the damaged area is identical with the axial position of the simulated recirculation bubble, the eccentricity of the damage is probably given by some production tolerances.

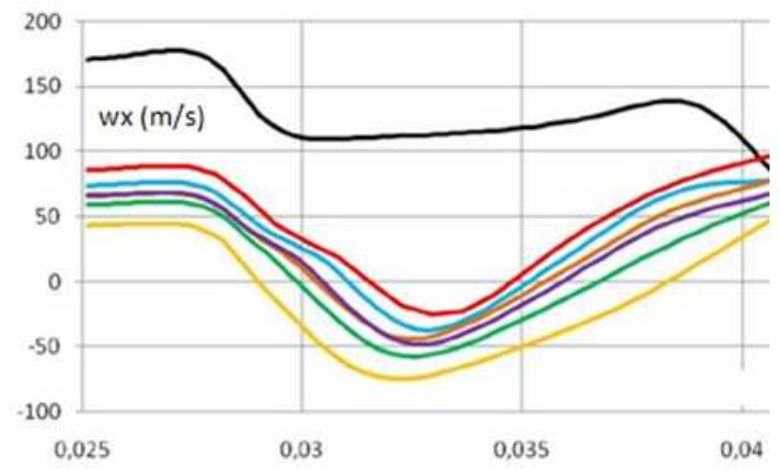

Figure 3-12. Axial velocity profiles in ejectors of cylindrical mixing tubes

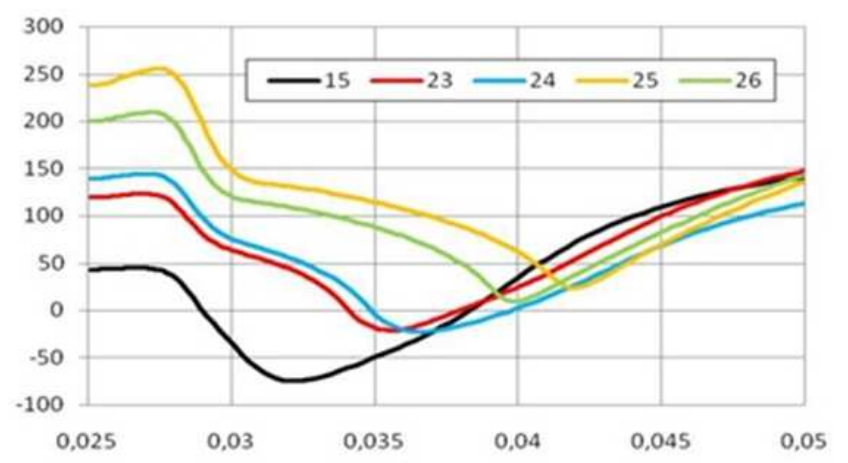

Figure 3-13. Axial velocity profiles in ejectors of divergent mixing tubes

Using several changes of the inner shape of the cylindrical mixing tube, several various velocity profiles were reached, together with tensile force profile, etc. From many results are in Fig. 3-12 presented velocity profiles for various shapes of long cylindrical mixing tubes (cases No. 14-18) for the comparison with the short one. Such a short mixing tube has not any backflow area, but its total tensile force is smaller.

The next cases (signed as No. 23-25) deal with shaped 
diffusing transition in the mixing tube, in comparison with one of the previous cylindrical mixing tubes (No. 15). Fig. 3-13 shows that using a suitable shaping it is possible to suppress the backflow (see the cases No. 25 and 26) - the values of the axial velocity are positive, only.

\subsection{Interaction of Flying Weft with Surroundings}

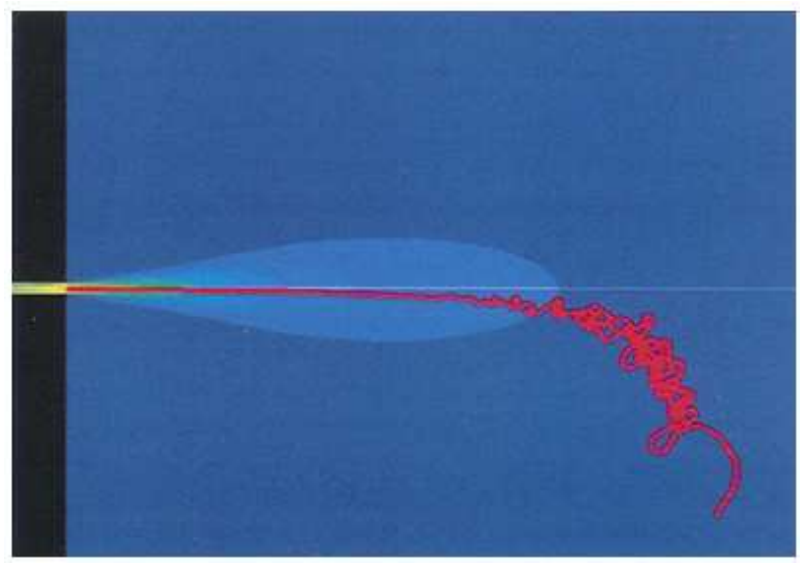

Figure 3-14. The shape of flying weft yarn in air flow and interaction with surroundings

The complicated case of mutual fluid-structure interaction between flying weft yarn, the driving air flow and stationary surrounding was solved in the collaboration with [23]. For this simulation the real textile material was replaced by smooth cylinder, whose diameter, density and elasticity modulus are responding to the reality. The Fig. 3-14 shows the area of the overcritical flow just after the mouth of the main nozzle, working on the principle of an ejector, together with transported weft yarns.

The results are interesting and are corresponding with the reality. At the action beginning, when in the flow from the nozzle mouth it is inserted the short weft tip, only, the weft shape is straight. With an increased distance from the nozzle mouth, the effect of the outgoing air flow is ceasing and the influence of both surrounding resistance and gravity, too, is relatively increasing. Fig. 3-14 presents the end of the observed time period when the tip of the transported weft is practically stopped, due to the continuing weft feeding, the primarily straight shape is crinkled and due to gravitation, the material is falling down from the flow area. In the reality, such an adverse situation is prevented by a serial of so-called auxiliary nozzles, which deliver into the flow axis the necessary kinetic energy, dissipating into the surroundings and in such a way, the axial velocity is preserved at the value necessary for the weft yarn transport along the whole weaving width in time and in a desired relatively straight shape. In general, simulation of such an interaction is very complicated.

\section{References}

[1] K. Adamek,"Conditions for High-quality and Reliable Air Jet Weft Insertion", Vlákna a textil (Fibers and textiles - in Czech) 5(3), 123-130 (1998), ISSN 1335-0617.
[2] K. Adamek, "Conditions for High-quality and Reliable Air Jet Weft Insertion", 2nd int. conf. Novelties in weaving research, TU Liberec, 1998 (EU project Copernicus).

[3] K. Adamek, "Numerical modelling the air flow in parts of air jet loom", CAMES (Comput. Assist. Mech. and Eng. Sciences), special issue, 6, 251-263, 1999, Polish Academy of Sciences Warsaw.

[4] K. Adamek, "Numerical modelling the air flow in parts of air jet loom", 8th int. conf. on numer. math. and comput. mech. (NMCM98), University of Miskolc, Hungary, 1998.

[5] J. Pelant, K. Adamek, "Boundary value conditions for some 3D axis-symmetrical flow", Proc. ECCOMAS CFD 2001, University of Wales, Swansea, 2001, ISBN 0-905091-11-6.

[6] K. Adamek, "Numerical modelling of air flow in air jet weaving system", Internat. J. Polymeric. Mater., 2000, Vol. 47, pp. 613-623, ISSN ....

[7] K. Adamek, "Numerical modelling of air flow in air jet weaving system", 32nd internat. symp. on novelties in textiles, University of Ljubljana, 1998, ISBN 961-6045-11-3.

[8] K. Adamek, "Numerical modelling of the flow effect on the weft during air jet weaving", VII. Internat. Congress on the Theory of Machines and Mechanisms, TU Liberec, 1996, ISBN 80-7083-198-7 (in Czech).

[9] K. Adamek, "Flow description in the air jet weaving system by FVM", Quo vadis textrinum, NMC Zilina, Strbske Pleso, 1996, ISBN 80-856655-06-3 (in Czech).

[10] K. Adamek, "Experience with FVM in Research of Air Jet Weaving System", Computer Modeling and Simulation in Engineering (CMSE), Vol. 1, Sage Science Press, 1996.

[11] K. Adamek, "Beschreibung der Webduesenstroemung mit FVM", 5. Chemnitzer Textilmaschinen-Tagung, TU Chemnitz, 1995.

[12] K. Adamek, "Design of parts for air jet weft insertion by FVM", Int. Conf. Young textile science, TU Liberec, 1995.

[13] K. Adamek, "Flow description in the air jet weaving system by FVM", Xth. sci. conf. Applic. of exp. meth. in fluid mech., VSDS Zilina, Strba, 1995 (in Czech).

[14] K. Adamek, "Le tissage a jet d'air", seminaire IMST, Univ. Marseille, 1994.

[15] J. Kolar, K. Adamek, V. Dvorak, "Aeroacoustic of jet induced noise", Conference of fluid- and thermomechanics departments on technical universities, 2009 (in Czech).

[16] J. Kolar, K. Adamek, "Aeroacoustic of simple shaped nozzles", EFM 2009 (Exp. Fluid Mech.), TU Liberec, Jicin, 2009, ISBN 978-80-7372-538-9.

[17] J. Simak, "Aeroacoustic analysis of circular nozzle", Transfer, VZLÚ Praha, No. 15, vol. 6, 2011. ISSN 1801-9315 (in Czech).

[18] K. Adamek, "Air flows in nozzles of air jet looms", VIth sci. conf. Applic. of exp. meth. in fluid mech. VSDS Zilina, Jasna, 1987 (in Czech).

[19] K. Adamek, "Applications of numerical flow modeling in designer's practice", XIVth int. conf. Applic. of exp. and numer meth. in fluid mech., ZU Zilina, 2004. ISBN 80-8070-234-9 (in Czech). 
[20] V. Tesar, "Reversation paradox", Acta technica. ČVUT Praha, 1984 (in Czech).

[21] K. Adamek, J. Pelant, "Reversation flow in ejector", XVIIth sci. conf. Applic. of exp. and numer. meth. in fluid mech. ZU Zilina, Bojnice 2010. ISBN 978-80-554-0189-8 (in Czech).
[22] J. Kolar, K. Adamek, J. Pelant, "Reversation flow in an ejector", Strutex, TU Liberec, 2010. ISBN 978-80-7372-664-5.

[23] S. Gramsch, D. Hietel, "Fadenschuss mittels Luftstrom", report of the Fraunhofer ITWM Kaiserslautern 